retrospectively. Once dispensed, TTOs are logged 'off' and sent by pneumatic chute system direct to the ward. This log and the hospital electronic prescribing system store relevant data including the time a TTO is written, dispensed and the patient discharged.

Results A total of 65 TTOs were dispensed in the pharmacy. (Others are prepared in a satellite unit, not included in this study). Only 18\% were prescribed more than 24 hours before discharge. Writing of TTOs clustered around $11 \mathrm{am}-4 \mathrm{pm}$ whereas patient discharges were around $12-1 \mathrm{pm}$ and $2-6 \mathrm{pm}$. Nearly $90 \%$ of TTOs were ready within 2 hours of the prescription being written. The average time from writing a TTO to the patient's discharge was 2.5 hours. The average dispensing time per patient was 1.2 hours. The Pharmacy element accounted for less than half the time patients were waiting for TTOs.

Conclusions The perception that dispensing of TTOs is responsible for significant delays in patient discharge is unfounded. There is a lag time between TTOs being ready and the patient going home which merits further investigation. The clustering of TTO writing infers that very few are written until the morning ward rounds are finished. Options are being explored to encourage earlier writing times such as including TTO-transcribing pharmacists on consultants' rounds.

No conflict of interest.

\section{DSL-008 DRUG SHORTAGES IN THE NETHERLANDS: MONITORED BY FARMANCO}

doi:10.1136/ejhpharm-2013-000276.251

DJ Postma, LM de Wolf. Royal Dutch Pharmacists Association, Scientific Institute for Dutch Pharmacists, Den Haag, The Netherlands

Background Internationally, drug shortages cause increasing concern. For patients it may impose a significant effect on their safe use of medicines. For pharmacists it is time-consuming to get trustworthy information.

Purpose With a central approach on the investigation of drug shortages, pharmacists get reliable and up-to-date information. Besides, solutions can be suggested. If there is a shortage of a necessary drug, proper action can be taken by all pharmacies.

With the data, trends in drug shortages can be signalled.

Materials and Methods In 2004 the Royal Dutch Pharmacists Association (KNMP) launched the website Farmanco: www. farmanco.knmp.nl. It provides pharmacists with up-to-date information on drug shortages in The Netherlands. Drug shortages are reported early and proper action can be taken. It provides information about the cause and duration of the shortage and a possible solution such as substitution or a pharmaceutical alternative.

Farmanco data from 2004 till 2011 were analysed to get an overview of the scale of the problem and more insight into the causes and solutions.

Results Through the years, the Farmanco website has become relevant to all concerned parties for up-to-date information. Visits to the website have increased to about 600 visitors on a weekday.

From 2004 till 2011 the Farmanco website published information on more than 1400 products.

Drug shortages have increased in frequency from 91 reported shortages in 2004 up to 242 in 2011.

The duration of a shortage has increased from 139 days (2004) to 254 days (2010).

Temporarily shortages are mainly caused by production problems $(52 \%)$, whereas permanent shortages usually have an economic reason $(69 \%)$.

The solutions have mainly been substitution (62\%), a pharmaceutical alternative $(25 \%)$ or pharmaceutical compounding $(2 \%)$. In $1 \%$ of the cases a solution was impossible.
Conclusions Farmanco gives pharmacists up-to-date information on drug shortages in The Netherlands.

Finally, trends in drug shortages can be signalled.

No conflict of interest.

\section{DSL-009 DRUG SHORTAGES: THE CHALLENGE OF IMPORTING}

doi:10.1136/ejhpharm-2013-000276.252

C Sousa. Hospital de Faro E.P.E. , Pharmacy, Faro, Portugal

Background Drug shortage has been reported since several years but has recently reached critical levels. Shortage occurs not only in Europe but worldwide, in all healthcare practise settings and affects potentially all drug classes, raw materials and medical devices. This combination of factors leads undoubtedly to medication overpricing and higher costs to the healthcare system, suboptimal clinical care, more medication errors and adverse events and the loss of patients' lives. Rational and effective procurement of medicines in foreign countries can be a challenge for hospital pharmacists.

Purpose The objective is to present a framework on medicines importation, with a special focus on European countries.

Materials and Methods Web search on governmental healthcare institutions (i.e. medicines' agencies), wholesalers, manufactures and other legal suppliers. This work was designed considering the Portuguese drug shortage.

Results A standard operation methodology was designed for searching for new suppliers for special medicines, not marketed or sold out. Search methodology on medicines' agencies is presented. A short framework for suppliers was filled considering regulatory issues, current good manufacturing practises, place in the drug supply chain, logistics, packaging, pricing, taxes, expedition costs and payment conditions. Web links to suppliers' websites are included. Conclusions Importation of medicines at the hospital level is more often part of the daily tasks of pharmacists. When treating critical health conditions, shortages in essential medicines can cause disruptions in patients' safety and quality of pharmacological treatment.

No conflict of interest.

\section{DSL-010 ECONOMIC IMPACT OF AUTOMATED DRUG DISPENSING SYSTEMS IMPLEMENTATION}

doi:10.1136/ejhpharm-2013-000276.253

A Linares-Alarcon, A Luna-Higuera, M Toca-Muñoz, MA Rosado Souviron, IM MuñozCastillo. HRU Carlos Haya, Pharmacy, Malaga, Spain

Background The distribution, management and control of drug stocks in clinical units is a responsibility of the pharmacy department, but this control is difficult to perform manually, resulting in a loss of important information about drug use.

Purpose To analyse the economic impact of automated drug dispensing systems (ADSs) implemented in the Intensive Care Unit (ICU) and the emergency department (ED).

Materials and Methods A total of 5 Omnicell cabinets were installed in August 2008: 3 in ICU and 2 in ED. The average cost of implementation for each one was about 60,000 euros. Nevertheless, the Hospital did not have to invest in them since they were donated by a national foundation.

The ICU is comprised of a total of 42 dedicated critical care beds located in 3 different modules, and ED has 2 modules with a total of 22 beds and 9 chairs.

The ADSs are connected to hospital admission software and to the pharmacy management software.

Medication costs in ICU and ED were examined, comparing one year prior to installation with the years after implantation of the ADS. These data were obtained from the management software of the pharmacy department. 